\title{
Motion of Polygonal Prisms Down Inclined Planes
}

AUTHORS: Liheng Chen ${ }^{1}$ and William Wei ${ }^{1}$

SCHOOL: 1. University of British Columbia, Vancouver, British Columbia, Canada

\section{EDITOR'S NOTE:}

The following is a project nominated for publication by the administrators behind Science One - a first-year science program at the University of British Columbia. Science One is an integrated program, where students have a chance to complete a research project in their second term.

\section{Abstract}

An experiment was carried out to determine the rolling times of square, hexagonal and circular prisms down inclined ramps of various angles. Each roll was recorded on video and rolling times were determined by counting frames. A model was created to predict the times of descent for $n$-sided prisms under the assumption of persistent contact between prism and ramp. A second model was created without this assumption. Animations of the prisms' motion were created according to the models. Predicted times of the models approached experimental times for polygonal prisms as the number of sides increased. Notable properties of the rolling motions are discussed, and reasons for discrepancies between models and results are addressed.

\section{Key Words}

Polygonal Prisms, Inclined Plane, Restitution Coefficient, Modelling

\section{Introduction}

Rotational and collisional interactions underlie the mechanics of systems that span a vast range of scales, from the rolling of pencils across a desk to the falling of boulders in a landslide. A rolling prism demonstrates inelastic collisions between its faces and the ramp surface. Between collisions, the prism touches the ramp along a single edge and rotates due to gravity. At greater angular speeds, the prism loses contact with the ramp. The prism is expected to reach a terminal velocity since a proportion of kinetic energy is lost in each collision [1]. An initial "walking model" was developed, which assumes contact between the prism and the ramp at all times. A more sophisticated "running model" was developed since the prism was observed to lose contact with the ramp in the experiment. Rolling times of the prisms were measured and were shown to depend on properties of the prisms and the angle of the ramp.

\section{Materials and Methods}

The prisms and the ramp consist of solid wood. The prisms' masses were determined using a top-loading balance and dimensions were measured using a caliper. The average radius $\bar{r}$ is the mean distance between the center and corners of each face, as shown in Table 1.

Table 1: Prisms' mass and dimensions, in which $\mathrm{u}[x]$ denotes the uncertainty of $x$.

\begin{tabular}{|c|c|c|c|c|}
\hline & mass $(\mathrm{kg})$ & $\mathrm{u}[\mathrm{mass}](\mathrm{kg})$ & $\bar{r}(\mathrm{~m})$ & $\mathrm{u}[\bar{r}](\mathrm{m})$ \\
\hline Square Prism & 0.02520 & 0.00003 & 0.01733 & 0.00005 \\
\hline Hexagonal Prism & 0.02800 & 0.00003 & 0.01413 & 0.00006 \\
\hline Circular Cylinder & 0.02330 & 0.00003 & 0.0119 & 0.0003 \\
\hline
\end{tabular}

Sandpaper of 180 grit was glued to the ramp to prevent sliding. The length of the ramp was measured to be $0.2790 \mathrm{~m}$. The inclination angle $\theta$ was determined trigonometrically by adjusting the vertical distance between the ground and a reference point on the ramp. The static friction coefficient $\mu_{f}$ between the sandpaper and the prisms was determined to be 0.923 by finding the maximum inclination angle such that the prism remains stationary and does not slide. Prisms were rolled with one corner touching the top edge of the sandpaper and released at a starting angle $\varphi_{0 i}$, defined as the angle between the vertical line through the contact corner and the radius segment of the corner, as shown in Figure 1. If the starting position is close to equilibrium, small variations in $\varphi_{0 i}$ can cause large differences in rolling times. Starting angles of around $5^{\circ}$ were marked on the prisms and exact starting angles were determined trigonometrically. The exact angles are presented in Table 2.

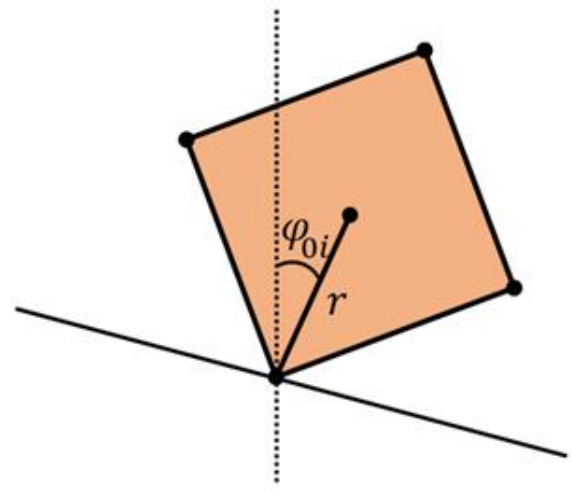

Figure 1: Illustration of $\varphi_{0 i}$ and $r$. 
Table 2: The initial starting angle $\varphi_{0 i}$ and its uncertainty $\mathrm{u}\left[\varphi_{0 i}\right]$ at which the prisms are released.

\begin{tabular}{|c|c|c|}
\hline & $\varphi_{0 i}(\mathrm{rad})$ & $\mathrm{u}\left[\varphi_{0 i}\right](\mathrm{rad})$ \\
\hline Square Prism & 0.08085 & 0.00001 \\
\hline Hexagonal Prism & 0.11350 & 0.00003 \\
\hline Circular Cylinder & $\theta$ & - \\
\hline
\end{tabular}

Each roll was filmed and frames were counted using QuickTime to determine the time between the prism's release and the instant when the prism's 3D center of mass is directly above the lower edge of the sandpaper. Frame numbers were converted to seconds using the phone's recording rate of 240.0 frames/second. Trials were discarded if the prism rolled sideways off the sandpaper as a result of imperfect release. The proportion of energy lost in face-on collisions is accounted by the restitution coefficient $e_{F}$, which is defined as the ratio of the angular speed right after a collision over the angular speed right before the collision. It is approximated by carefully releasing the prism near equilibrium and observing whether the prism reaches its next equilibrium after one collision. The equilibrium is defined as the position at which the prism balances on its edge. The ramp is adjusted until a critical inclination angle $\theta_{c}$ is reached where upon release, the prism just reaches its next equilibrium. For an $n$ sided polygonal prism,

$$
e_{F}=\frac{\sqrt{1-\cos \left(\theta_{c}-\frac{\pi}{n}\right)}}{\sqrt{1-\cos \left(\theta_{c}+\frac{\pi}{n}\right)}} .
$$

Since square and hexagonal prisms are not perfectly shaped, $e_{F}$ was measured for each face of the prism and an average was obtained as $\overline{e_{F}}$. When a "running" prism regains contact with the ramp, its energy loss is described by the edge-on coefficient $e_{E}$, which has not been determined in the experiment and is assumed to have the same value as $\overline{e_{F}}$ in the models.

\section{Models}

The motion of a regular $n$-sided polygonal prism down an inclined plane is simplified by considering the movement of a two-dimensional polygon down a linear ramp. Given a release angle $\varphi_{i}$ and an angular speed $\omega_{i}$, the walking model predicts the angle $\varphi_{i+1}$ after a small time interval $\Delta t$, by assuming a constant angular speed over this interval. The angular speed $\omega_{i+1}$ is derived from energy conservation, giving

$$
\omega_{i+1}=\sqrt{\omega_{i}^{2}+\frac{2 M g r}{I}\left(\cos \left(\varphi_{i}\right)-\cos \left(\varphi_{i+1}\right)\right)},
$$

where $M$ is the mass of the prism and $I$ is its moment of inertia about an edge, given by

$$
I=\frac{M r^{2}}{6}\left(2 \cos ^{2}\left(\frac{\pi}{n}\right)+7\right)[2] .
$$

With initial $\varphi_{0}$ and $\omega_{0}$, prognosticated values for $\varphi_{i}$ and $\omega_{i}$ are determined using Euler integration. When $\varphi=\theta+\frac{\pi}{n}$, a face-on collision occurs and $\varphi$ is reset to $\theta-\frac{\pi}{n}$ as the corner of contact switches, where $n$ is the number of sides of the prism. To account for energy loss, the angular speed right after a collision is given by multiplying the angular speed immediately before the collision with $e_{F}$. The horizontal displacement of the center of mass, $\Delta x$, is tracked until the prism reaches the end of the ramp. The cylinder model uses $n=1000$ and assumes $e_{F}=1$ since negligible energy should be lost during the rolling motion of the cylinder. Approximating a cylinder with $n=1000$ is sufficient since the results agreed to 4 significant figures when $n=10000$ is used.

The running model, unlike the walking model, accounts for prisms' loss of contact with the ramp. When $\omega>\sqrt{\frac{g \cos (\varphi)}{r}}$, the prism loses contact since the centripetal acceleration about the corner of rotation overcomes the component of gravity along the effective radius [3]. At the instant of losing contact, the horizontal and vertical speeds of the center of mass are respectively $v_{x 0}=$ $\omega_{0} r \cos \left(\varphi_{0}\right)$ and $v_{y 0}=-\omega_{0} r \sin \left(\varphi_{0}\right)$, where $\omega_{0}$ is the angular speed and $\varphi_{0}$ is the angle at the moment of losing contact. The height of each corner above the ramp as a function of time $H_{m}(t)$ is described by

$H_{m}(t)=r \cos \left(\varphi_{0}\right)+r \sin \left(\varphi_{0}\right) \tan (\theta)+v_{y 0} t-\frac{1}{2} g t^{2}+$

$\tan (\theta) v_{x 0} t+r \cos \left(\mu_{m}\right) \tan (\theta)-r \sin \left(\mu_{m}\right)$,

where $t$ is the elapsed time since losing contact, $m$ is the index of each corner, and $\mu_{m}$ is the clockwise angular distance from the horizontal. The last corner in contact with the ramp is indexed 0 and subsequent corners are indexed counter-clockwise as shown in Figure 2.

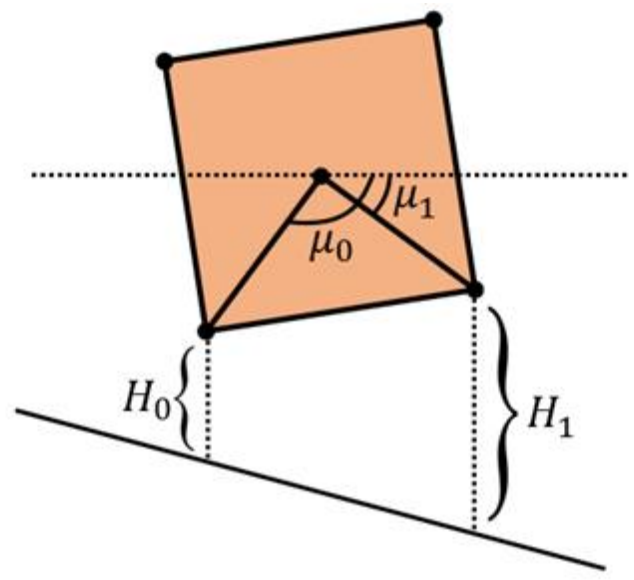

Figure 2: Illustration of $H$ and $\mu$. 

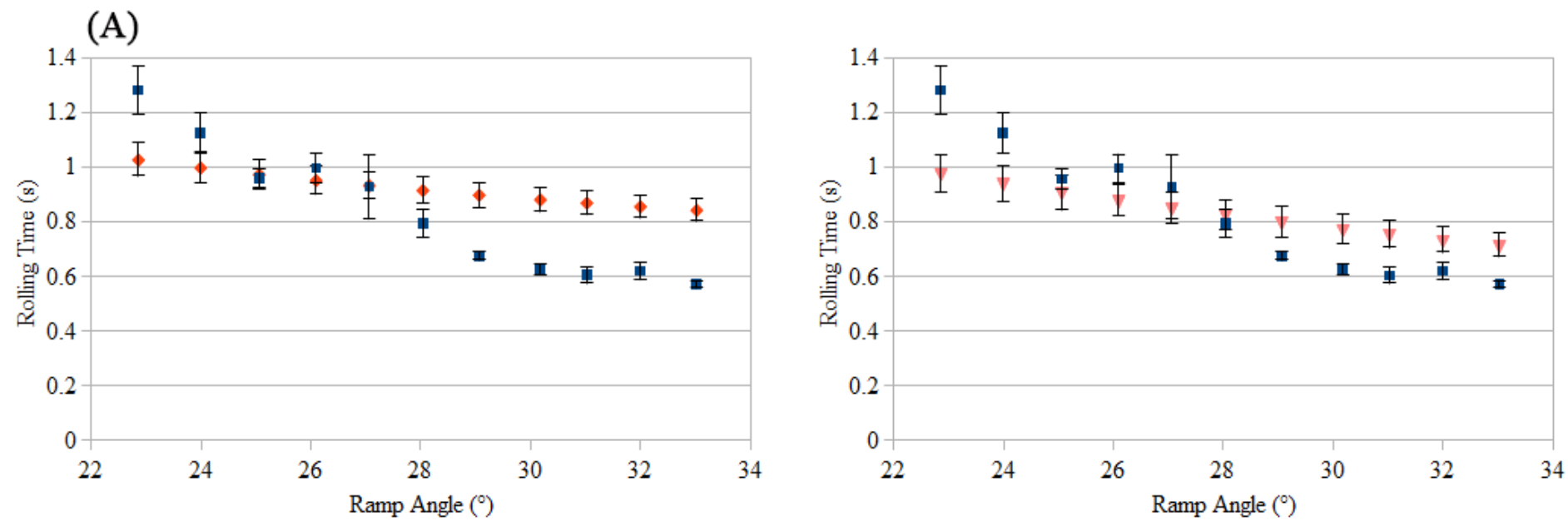

(B)
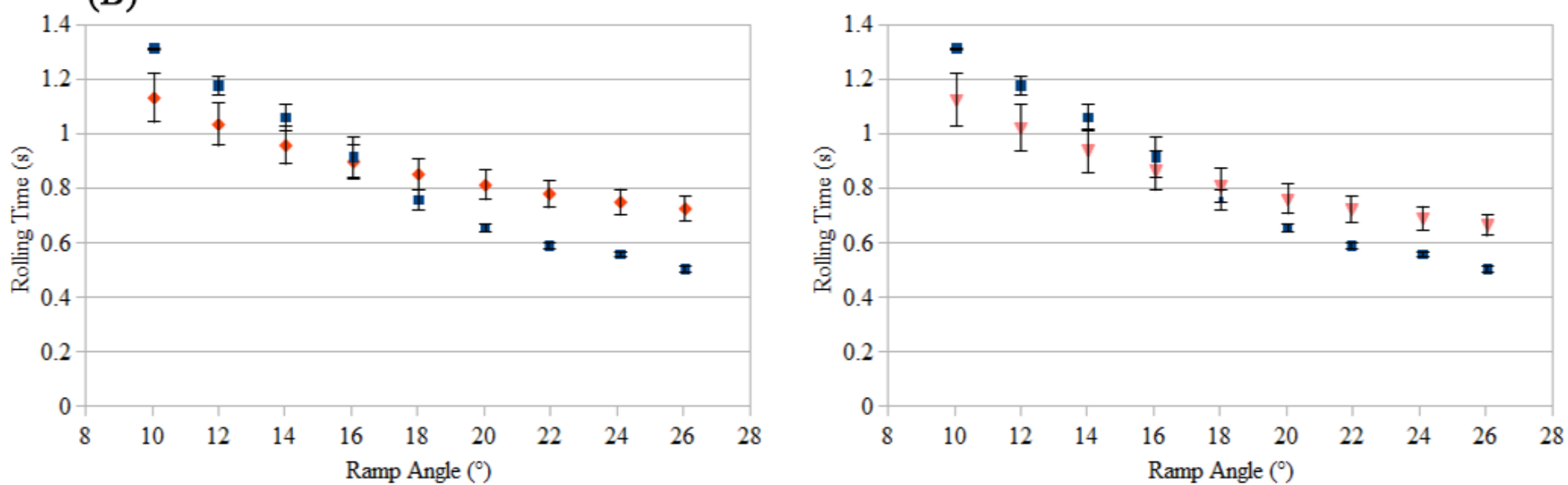

(C)
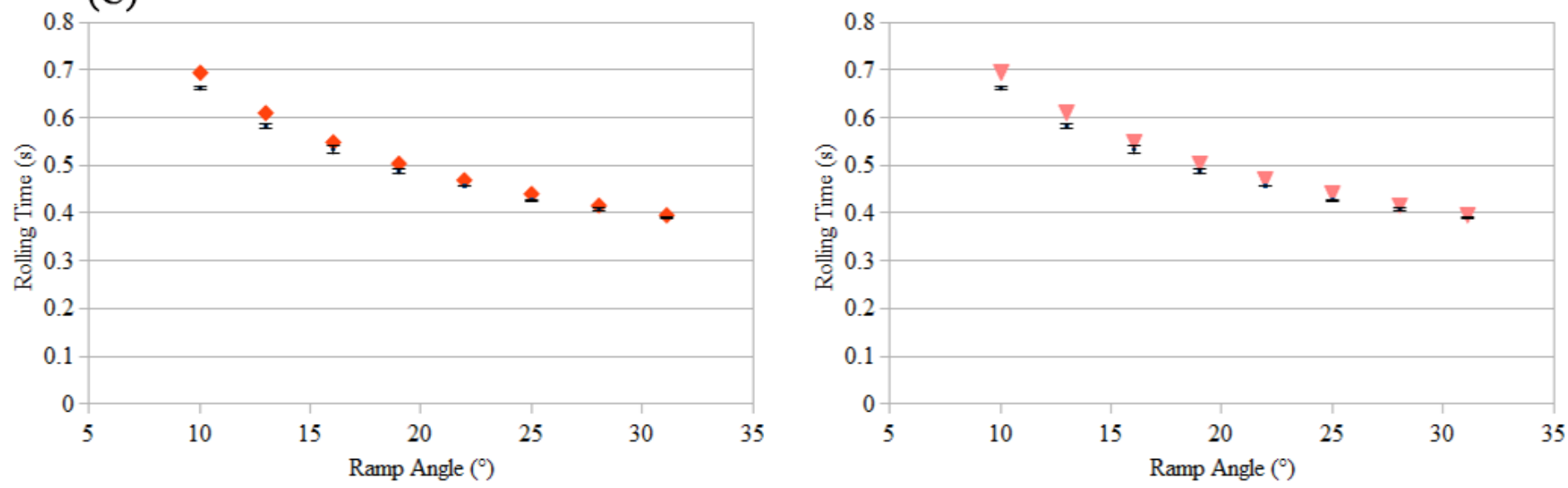

Figure 3: Comparison of model and experiment times.

(A) Rolling times for the square prism at various ramp angles.

(B) Rolling times for the hexagonal prism at various ramp angles.

(C) Rolling times for the cylinder at various ramp angles.

Model-predicted rolling times are determined by inputting the corresponding $\theta, l, \overline{e_{F}}$ for each roll. Each experimental data point is the average of four or five trials. Error bars of experiment represent standard mean error; error bars for model represent $68 \%$ confid ence intervals. 
The angular distance for each corner is given by

$$
\mu_{m}=\frac{\pi}{2}+\varphi_{0}-m \frac{2 \pi}{n}+\omega_{0} t .
$$

$H_{m}(t)$ is determined by updating $\mu_{m}$ under the assumption of constant angular speed through the air. The horizontal displacement of the center of mass is updated accordingly until any $H_{m}$ reaches zero and the prism regains contact. Immediately, the new initial walking angle $\varphi_{0 r}$ and angular speed $\omega_{0 r}$ are

$$
\omega_{0 r}=\sqrt{\frac{2}{I} e_{E}^{2}\left(\frac{1}{2} M v_{x 0}{ }^{2}+\frac{1}{2} M\left(v_{y 0}-g t_{f}\right)^{2}+\frac{1}{2} I_{c} \omega_{0}^{2}\right)} .
$$

where $t_{f}$ is the total flying time, and $I_{c}$ is the moment of inertia about the central axis of the prism, obtained by reverse application of the Parallel Axis Theorem to Equation (3). If $\omega_{0 r}$ is greater than the critical walking speed, the running model starts again; otherwise, the prism continues with the walking model until the next moment of losing contact.

\section{Results and Discussion}

Rolling times for models and experiments are shown in Figure 3 , and the average restitution coefficients determined for square and hexagonal prisms are shown in Table 3.

Table 3: $\overline{e_{F}}$ for each prism.

\begin{tabular}{|c|c|c|}
\hline & $\overline{e_{F}}$ & $\mathrm{u}\left[\overline{e_{F}}\right]$ \\
\hline Square prism & 0.656 & 0.030 \\
\hline Hexagonal prism & 0.856 & 0.026 \\
\hline
\end{tabular}

The uncertainties in radius, mass, rolling length, starting angle, and $\overline{e_{F}}$ all contribute to errors in modelling; however, the relative uncertainty in $\overline{e_{F}}$ is an order of magnitude greater than the uncertainties in the other values. Hence, the error in model times are determined by inputting the upper and lower bounds of $\overline{e_{F}}$, and taking other uncertainties as negligible. Error bars are excluded from the cylinder model since it is assumed that $e_{F}=1$.

Root mean square deviation (RMSD) measures the differences between model and experiment. Running models have lower RMSD than walking models and are therefore more accurate, as shown in Table 4. RMSD decreases when $n$ increases, which suggests that both models may better predict rolling times for larger $n$.

Table 4: RMSD.

\begin{tabular}{|c|c|c|c|}
\hline & Square prism & Hexagonal prism & Circular prism \\
\hline Walking model & 0.1914 & 0.1559 & 0.018580 \\
\hline Running model & 0.1489 & 0.1295 & 0.018577 \\
\hline
\end{tabular}

In general, the running model agrees with the walking model for small $\theta$, but predicts rolling times better than the walking models for larger $\theta$. The overall trend of the cylinder model agrees with the experiment; however, most of the predicted values lie outside the experiment's error bars. This may be due to the wood cylinder's nonuniform density, which could result in a smaller moment of inertia. The running model is more effective for smaller $n$, yet both models make more accurate predictions as $n$ increases.

The underestimation of rolling times at smaller $\theta$ may result from double bouncing. A double bounce consists of a face-on collision followed immediately by an edge-on collision, as shown in Figure 4 . Both models only capture the dynamics of single bounces; however, additional energy is lost in double bouncing. This lower energy profile translates to greater rolling times in experiments. In addition, the walking model overestimates rolling times for large $\theta$ since it does not account for the running motion that prisms exhibit in experiments. The overestimation in the running model for large $\theta$ may be due to equating $e_{E}$ with $e_{F}$ in the model. An edge-on collision typically experiences a smaller energy loss, as shown by the maximum rebound heights in Figure 5. As such, $e_{E}$ must be greater than $e_{F}$. Furthermore, $e_{E}$ is difficult to be experimentally quantified. Edge-on collisions do not always occur perpendicularly, so energy loss and $e_{E}$ may vary with collision angle.

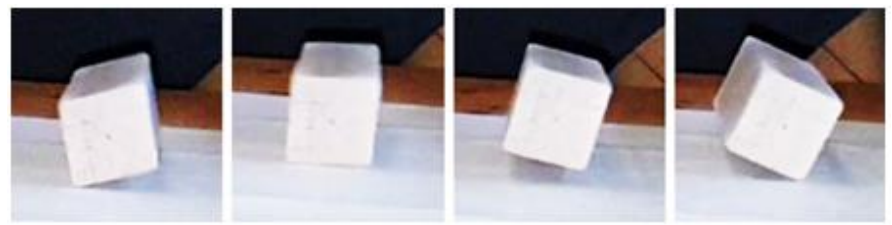

Figure 4: Snapshots of double bouncing with $\theta \approx 23^{\circ}$, in chronological order from left to right.

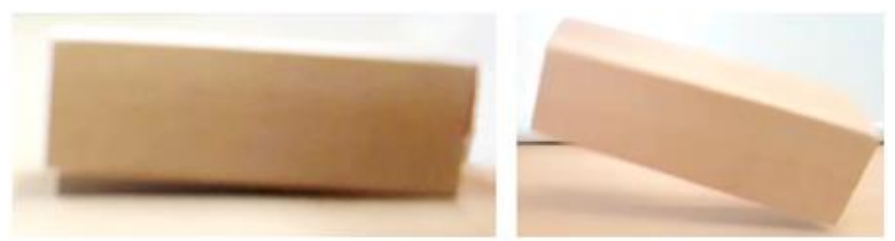

Figure 5: Maximum rebound for a square prism in a face-on collision (left) and edge-on collision (right) dropped from the same height.

Since the restitution coefficient is the largest source of error in the experiment, predicted rolling times for the walking model were plotted against varying $e_{F}$ to determine possible relationships. All other parameters were kept the same as in the walking model for the square prism. Rolling time decreases with greater restitution coefficient, as shown in Figure 6. For values of $e_{F}$ below 0.30 , the prism is unable to reach its next equilibrium position. A restitution coeffi- 
cient closer to one results in a more constant curve at the experimental angles, shown in Figure 7.

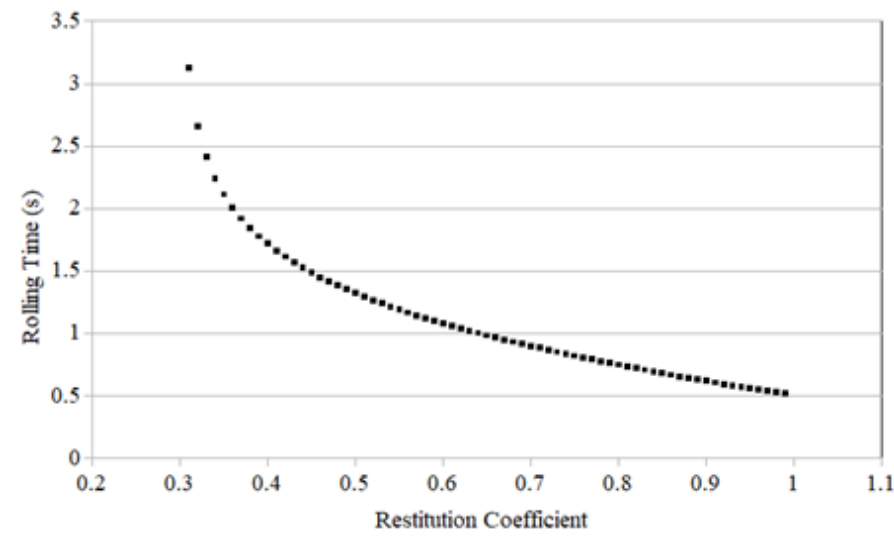

Figure 6: Model-predicted rolling times of square prism for various restitution coefficients $0.31 \leq e_{F} \leq 0.99$, with $l=0.279 \mathrm{~m}, \theta=$ $\underline{25^{\circ}}$

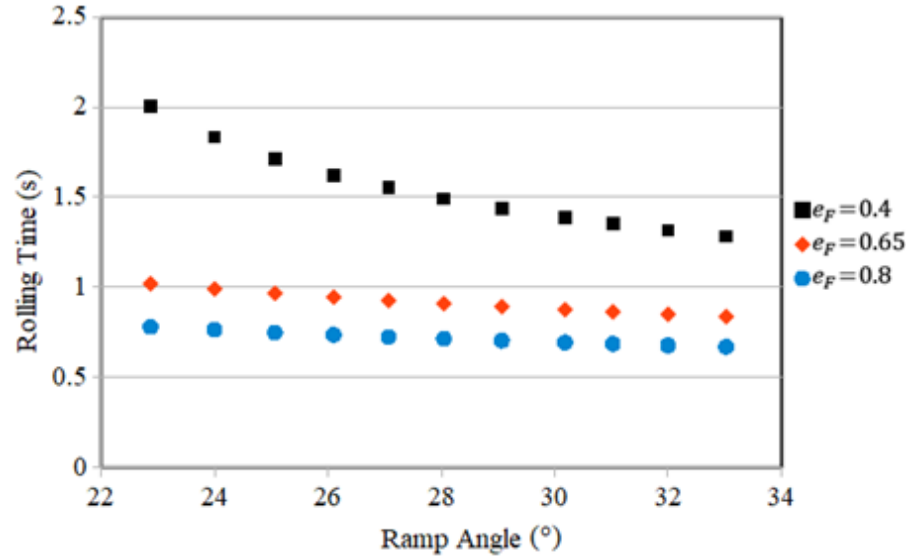

Figure 7: Model-predicted rolling times for the square prism at various ramp angles with $e_{F}=0.4,0.65,0.8 ; l=0.279 \mathrm{~m}$.

For $e_{F}<1$, the prism approaches a terminal velocity in the limit of large $t$. For the walking model, the kinetic energy $E_{k}$ of the prism after each collision plateaus to

$$
E_{k \infty}=2 \frac{e_{F}^{2}}{1-e_{F}^{2}} m g r \cdot \sin \left(\frac{\pi}{n}\right) \cdot \sin (\theta) .[1]
$$

The terminal angular speed $\omega_{\varphi \infty}$ for a specific position $\varphi$ is given by

$$
\omega_{\varphi \infty}{ }^{2}=\frac{2}{I}\left(E_{k \infty}+m g r\left(\cos \left(\theta-\frac{\pi}{n}\right)-\cos (\varphi)\right)\right) .
$$

The dependence of the terminal velocity on $e_{F}$ is shown in Figure 8. The plot is not perfectly linear since $\omega_{\varphi \infty}$ cannot be precisely reached with a finite ramp. The slope of the plot is reasonably close to $\frac{4}{I} m g r \cdot \sin \left(\frac{\pi}{4}\right) \cdot \sin (\theta)$, which is in agreement with calculations.

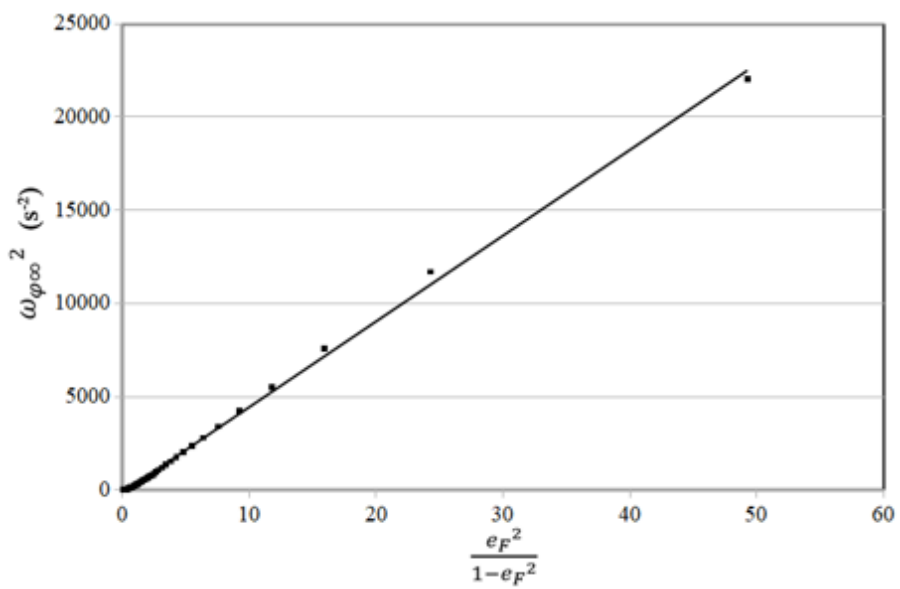

Figure 8: Relationship between terminal velocity and restitution coefficient for the square prism walking model, $0.31 \leq e_{F} \leq 0.99$. $\underline{l}=2.79 \mathrm{~m}, \theta=25^{\circ}$.

\section{Conclusion}

The rolling times of square, hexagonal and circular prisms at various inclination angles follow a trend similar to that obtained from the walking and running models. The cylinder had the strongest agreement with the models. The running model provides better predictions for the rolling times of square and hexagonal prisms, but the two models converge with sufficiently large $n$. Further investigation into $e_{E}$ and quantification of double-bounces will lead to more effective models.

\section{Acknowledgement}

We would like to thank our mentor Dr. Stephen Gustafson as well as Dr. James Charbonneau for their insight and guidance.

\section{References}

1. McDonald KT. Hexagonal pencil rolling on an inclined plane. Regular and Chaotic Dynamics. 2008;13(4):332-43.

2. Rektorys K. (1994). Survey of applicable mathematics. 2nd ed. Dordrecht: Kluwer Acad. Publ; 1994.

3. Rezaeezadeh A. Motion of a hexagonal pencil on an inclined plane. American Journal of Physics. 2009;77(5):401-6. 


\section{REVIEW}

\section{by Dr. Robert Raussendorf \\ Department of Physics and Astronomy, University of British Columbia}

The authors derive two mathematical models for $\mathrm{n}$-sided prisms rolling down an inclined plane, and test their predictions of roll times against experiment. The video footage from the experiments also allows studying individual collisions between prisms and plane in detail.

The agreement between theory and experiment is good, in particular for the significantly more involved "running model", but it is not perfect. For 4 and 6-sided prisms, the predicted roll times vary more weakly with the incline angle than the experimental data shows.

A small suggestion (optional) therefore is the following: The authors point out in Fig. 7 that lower restitution coefficients lead to steeper curves, and the question thus arises if better agreement between theory and experiment could be obtained by fitting the parameters $e_{\mathrm{E}}, e_{\mathrm{F}}$. Perhaps it is worth trying. A physical justification would be that the restitution coefficients could be velocity-dependent. The coefficient $e_{\mathrm{F}}$ is determined in a near-static experiment whereas the $e_{\mathrm{F}}$ that enters the simulation is at higher velocities.

I like the aspect that video footage is included in the figures, to illustrate the double-bounce.

Overall, this is substantial work, of high quality. The models for the rolling process are worked out in detail, and the resulting differential equations are integrated numerically. The predictions are compared with experiment, and match to a good degree of accuracy. The remaining discrepancies are analyzed, and possible refinements of the model are suggested to account for them. Therefore, I recommend this manuscript for publication in the STEM Fellowship Journal. 


\section{SUPPLEMENTAL DERIVATIONS}

\section{Derivation of Walking Model}

The instantaneous angular velocity is approximated by considering the mechanical energy of the polygon. At some instant $t_{i}$, the polygon has angle $\varphi_{i}$ and angular speed $\omega_{i}$, and has mechanical energy

$$
U_{i}=\frac{1}{2} I \omega_{i}^{2}+m g r \cos \varphi_{i}+C,
$$

where $I$ is the polygon's second area moment and $C$ is the gravitational potential energy that the prism has when its center of mass is vertically aligned with the corner of contact. At a time $\Delta t$ after this instant, the subsequent angle $\varphi_{i+1}$ can be approximated by Euler integration as

$$
\varphi_{i+1}=\varphi_{i}+\omega_{i} \Delta t
$$

This can be used to approximate the energy of the polygon at $t_{i+1}$ giving

$$
U_{i+1}=\frac{1}{2} I \omega_{i+1}{ }^{2}+m g r \cos \left(\varphi_{i}+\omega_{i} \Delta t\right)+C .
$$

Neglecting air drag, mechanical energy is conserved within one roll; this model assumes that sliding never occurs, so friction does not do work. As a result, $U_{i}=U_{i+1}$ and the subsequent angular speed can be approximated by

$$
\omega_{i+1}=\sqrt{\omega_{i}^{2}+\frac{2 m g r}{I}\left(\cos \varphi_{i}-\cos \left(\varphi_{i}+\omega_{i} \Delta t\right)\right)} .
$$

Eq (ii) and (iv) are used to calculate $\varphi$ and $\omega$ for subsequent instances.

\section{Derivation of Restitution Coefficient (Face-on Collision):}

Assuming that the experiment is ideal, a prism balanced at its equilibrium on a ramp inclined to $\theta$ would experience an infinitesimally small impulse and stop precisely at its next equilibrium. Before the collision, potential energy is fully converted to kinetic energy and

$$
m g\left(1-\cos \left(\theta+\frac{\pi}{n}\right)\right)=\frac{1}{2} I \omega_{i}^{2},
$$

where $n$ is the number of sides on the polygon and $\omega_{i}$ is the angular speed right before a collision. After the collision, kinetic energy is fully converted to potential energy, yielding

$$
\frac{1}{2} I \omega_{f}^{2}=m g\left(1-\cos \left(\theta-\frac{\pi}{n}\right)\right) .
$$

Given $e_{F} \equiv \frac{\omega_{f}}{\omega_{i}}$

$$
e_{F}=\frac{\sqrt{1-\cos \left(\theta-\frac{\pi}{n}\right)}}{\sqrt{1-\cos \left(\theta+\frac{\pi}{n}\right)}} .
$$




\section{Derivation of Running Model}

\section{Criterion of Losing Contact:}

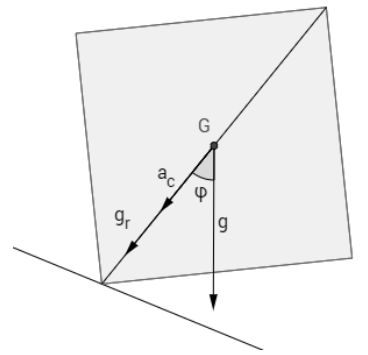

Figure I.

Centripetal acceleration: $a_{c}=\omega^{2} r$;

Gravitational component: $g_{r}=g \cos \varphi$.

When $a_{c}>g_{r}, \omega>\sqrt{\frac{g \cos \varphi}{r}}$ (in the experiment, $-\frac{\pi}{2}<\varphi<\frac{\pi}{2}$, and $\cos \varphi>0$ ).

\section{Initial Velocities}

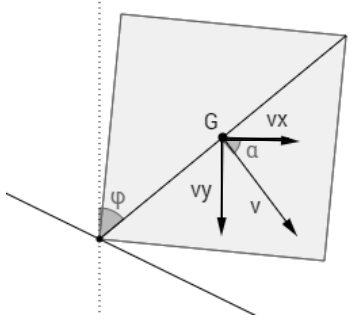

Figure II.

$\varphi$ in Figure II represents $\varphi_{0}$.

$$
a=\varphi_{0} \text {. }
$$$$
v_{x 0}=v \cos \varphi_{0}, v_{y 0}=-v \sin \varphi_{0} .
$$

Given $v=\omega r, v_{x 0}=\omega r \cos \varphi_{0}$ and $v_{y 0}=-\omega r \sin \varphi_{0}$. 


\section{Derivation of $\boldsymbol{H}(\boldsymbol{t})$ :}

$\mathrm{H}$ was broken down into components as shown in Figure III.

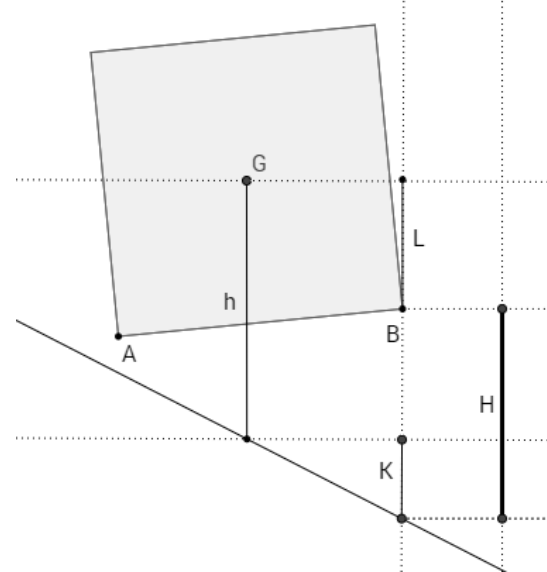

Figure III.

$\mathrm{H}=\mathrm{h}-\mathrm{L}+\mathrm{K}$.

$h_{0}, L_{0}$ and $K_{0}$ :

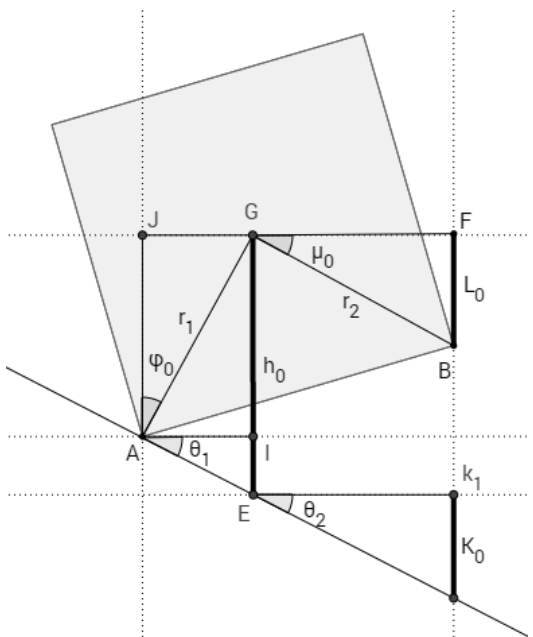

Figure IV.

$$
\begin{gathered}
\mathrm{L}_{0}=r \sin \left(\mu_{0}\right) . \\
K_{0}=\overline{E k_{1}} \tan (\theta)=\overline{G F} \tan (\theta)=r \cos \left(\mu_{0}\right) \tan (\theta) . \\
h_{0}=\overline{A J}+\overline{I E}=r \cos \left(\varphi_{0}\right)+\overline{A I} \tan (\theta)=r \cos \left(\varphi_{0}\right)+\overline{J G} \tan (\theta)=r \cos \left(\varphi_{0}\right)+ \\
r \sin \left(\varphi_{0}\right) \tan (\theta) . \\
\mathrm{H}_{0}=h_{0}-L_{0}+K_{0}, \\
\mathrm{H}_{0}=r \cos \left(\varphi_{0}\right)+r \sin \left(\varphi_{0}\right) \tan (\theta)+r \cos \left(\mu_{0}\right) \tan (\theta)-r \sin \left(\mu_{0}\right),
\end{gathered}
$$

where $\mu_{0}=\varphi_{0}$ (for the square, but more generally,

$$
\mu_{0}=\pi-\left(\frac{\pi}{2}-\varphi_{0}\right)-\frac{2 \pi}{n}=\frac{\pi}{2}-\frac{2 \pi}{n}+\varphi_{0}
$$

for any $n$-sided polygon). 


\section{$h(t), L(t)$ and $K(t)$ :}

Let $t$ be the time spent in the air and let $t=0$ be the time instant of losing contact.

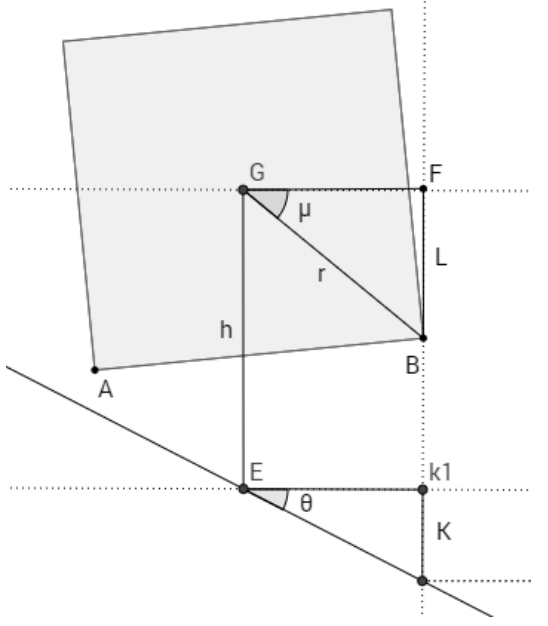

Figure $\mathbf{V}$.

Neglecting air resistance, $\omega_{0}$ and $v_{x 0}$ are constant while the polygon is in the air. As such,

$$
\begin{gathered}
\mu(t)=\mu_{0}+\omega_{0} t, \\
\mathrm{~L}(\mathrm{t})=r \sin (\mu), \\
\mathrm{K}(\mathrm{t})=r \cos (\mu) \tan (\theta) .
\end{gathered}
$$

When the square moves forward, $h(t)$ increases. the horizontal displacement of the square results in an increase in the vertical distance between point $\mathrm{G}$ and $\mathrm{E}$, which is equal to $v_{x 0} t \tan (\theta)$. In addition, the vertical displacement of an object with initial vertical velocity $v_{y 0}$ is given by $v_{y 0} t-\frac{1}{2} g t^{2}$. Combining the above,

Overall,

$$
\mathrm{h}(\mathrm{t})=h_{0}+v_{x 0} t \tan (\theta)+v_{y 0} t-\frac{1}{2} g t^{2} .
$$

$$
\begin{gathered}
\mathrm{H}(\mathrm{t})=r \cos \left(\varphi_{0}\right)+r \sin \left(\varphi_{0}\right) \tan (\theta)+v_{x 0} t \tan (\theta)+v_{y 0} t-\frac{1}{2} g t^{2}+r \cos (\mu) \tan (\theta)- \\
r \sin (\mu),
\end{gathered}
$$

Computationally, $\mathrm{H}(\mathrm{t})$ starts at $H_{0}$, and $t$ increases by $d t$, using Euler integration until $H(t)=0$. In essence, the program solves for $t$ when $H(t)=0$, and defines the first solution to be $t_{f}$. When the prism lands, it loses some kinetic energy modelled by the coefficient $e_{E}$. Immediately before the collision, the prism possesses kinetic energy $\mathrm{E}_{k}$. Immediately after the collision, the prism has kinetic energy $\mathrm{E}_{r}=e_{E}{ }^{2} \mathrm{E}_{k}$ (subscript $\mathrm{r}$ denotes "running"),

$$
\mathrm{E}_{r}=e_{E}^{2}\left(\frac{1}{2} m v_{x 0}^{2}+\frac{1}{2} m\left(v_{y 0}-g t_{f}\right)^{2}+\frac{1}{2} I_{c} \omega_{0}^{2}\right) .
$$

Immediately after the prism lands, the angular speed with respect to the corner of contact is $\omega_{r 0}=\sqrt{\frac{2}{I} \mathrm{E}_{r}}$. The angular position is redefined as $\varphi_{0 r}$ when the prism lands. The two possible cases are shown in figures VI.1 and VI.2. 


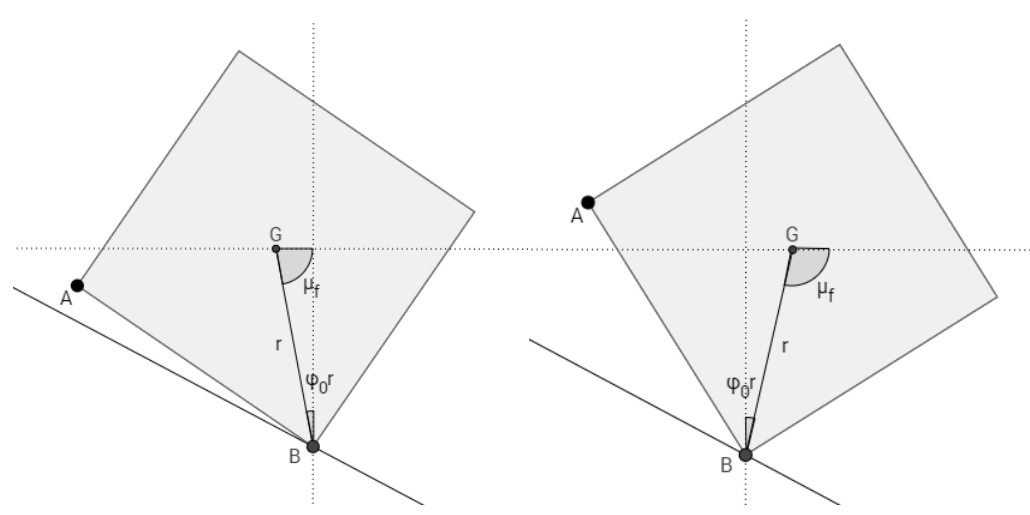

Figure VI.1, VI.2.

$\varphi_{0}$ was defined to be negative when the center of mass is behind the equilibrium, and positive otherwise. As such,

$$
\begin{gathered}
\varphi_{0 r}=-\left(\frac{\pi}{2}-\mu_{f}\right), \\
\text { where } \mu_{f}=\mu_{0}+\omega_{0} t_{f} .
\end{gathered}
$$

Right after landing, there are two possible scenarios. If $\omega_{0 r}>\sqrt{\frac{g \cos \varphi_{0 r}}{r}}$, the prism would immediately lose contact again; if $\omega_{0 r} \leq \sqrt{\frac{g \cos \varphi_{0 r}}{r}}$, the prism will roll until it loses contact again. In the first scenario, the prism would be modelled by the running model. In the second scenario, the walking model would be applied until the prism runs again. In the first scenario, it is possible that $\varphi_{0}$ would start negative, as shown in Figure VII;

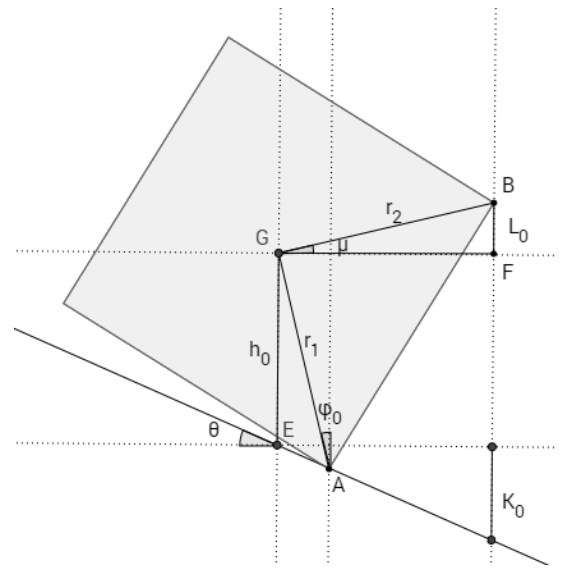

Figure VII.

however, all relevant equations still apply.

All the above equations hold true for any $n$-sided regular polygon. However, as discussed in paper, edge-skipping must be accounted for as follows. 


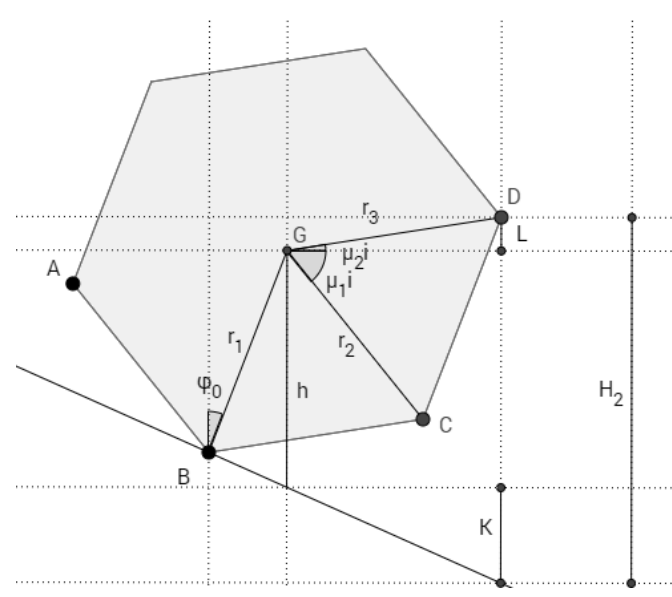

Figure VIII.

Define $\mu$ to be negative when the corner is above the horizontal line through G. For example, when $m=2, n=6$,

$$
\mu_{2, i}=\mu_{1, i}-\frac{\pi}{3}=\varphi_{0}-\frac{\pi}{6}
$$

More generally,

$$
\mu_{m}=\mu_{m i}+\omega_{0} t=\frac{\pi}{2}+\varphi_{0}-m \frac{2 \pi}{n}+\omega_{0} t
$$

Similarly, $H_{m}(t)=h-L+K$, that is,

$$
\begin{gathered}
H_{m}(t)=r \cos \left(\varphi_{0}\right)+r \sin \left(\varphi_{0}\right) \tan (\theta)+v_{y 0} t-\frac{1}{2} g t^{2}+v_{x 0} t * \tan \theta-r \sin \mu_{m}+ \\
r \cos \mu_{m} \tan \theta
\end{gathered}
$$

The landing angle $\varphi_{0 r}$ can be redefined as

$$
\varphi_{0 r}=-\frac{\pi}{2}+\mu_{m i}+\omega_{0} t_{f}
$$

where $t_{f}$ is the time when any $H_{m}$ reaches 0.

\section{Derivation of Uncertainty of Starting Angle}

The starting angle $\varphi_{0}$ is measured by drawing a right triangle with respect to $\varphi_{0}$, and measuring the opposite-side length $D$ and hypotenuse $l$ of the triangle with a caliper to obtain

$$
\varphi_{0}=\sin ^{-1}\left(\frac{D}{l}\right) \text {. }
$$

The uncertainty of $\varphi_{0}(D, l)$ is derived by manipulating the partial derivatives with respect to $D$ and $l$,

$$
\mathrm{u}\left[\varphi_{0}\right]=\sqrt{\left(u[D] \frac{d \varphi_{0}}{d D}\right)^{2}+\left(u[l] \frac{d \varphi_{0}}{d l}\right)^{2}}
$$




\section{Derivation of Terminal Velocity}

Let the kinetic energy gained before the first collision be $E_{0}$, and the kinetic energy right after $\mathrm{c}^{\text {th }}$ collision be $E_{k c}$. Recall $\Delta E$ is the kinetic energy gained between each collision.

$$
\begin{gathered}
E_{k 1}=E_{0} \cdot e_{F}{ }^{2}, \\
E_{k 2}=\left(E_{0} \cdot e_{F}{ }^{2}+\Delta E\right) e_{F}{ }^{2}, \\
E_{k 3}=\left(E_{0} \cdot e_{F}{ }^{2}+\Delta E \cdot e_{F}{ }^{2}+\Delta E\right) e_{F}{ }^{2}, \\
E_{k c}=E_{0} \cdot e_{F}{ }^{2 c}+\left(\Delta E \cdot\left(e_{F}{ }^{2}\right)^{c-1}+\Delta E \cdot\left(e_{F}{ }^{2}\right)^{c-2}+\cdots+\Delta E \cdot\left(e_{F}{ }^{2}\right) .\right. \\
\text { For } 0<e_{F}<1, E_{k c}=\Delta E \frac{e_{F}{ }^{2}}{1-e_{F}{ }^{2}} \text { when } \mathrm{c} \rightarrow \infty .
\end{gathered}
$$

$\Delta E$ is equal to the potential energy lost between each collision.

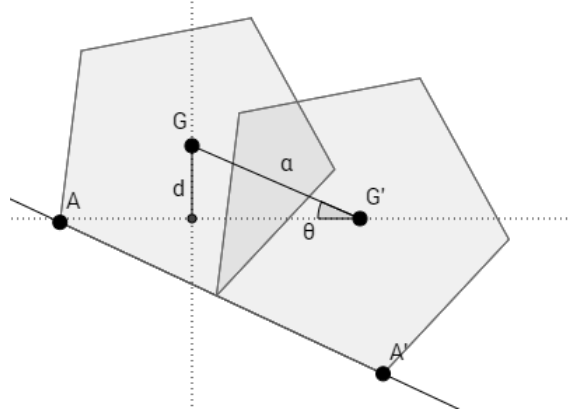

Figure IX

As shown in Figure IX,

$$
\begin{gathered}
\Delta E=m g d, \\
d=a \sin \theta, \\
a=2 r \sin \left(\frac{\pi}{n}\right), \\
\Delta E=m g 2 r \sin \left(\frac{\pi}{n}\right) \sin \theta .
\end{gathered}
$$

The term $m g r\left(\cos \left(\theta-\frac{\pi}{n}\right)-\cos \varphi\right)$ in Eq (16) represents the potential energy difference between the position right after collision and the position with $\varphi$, as shown in Figure $\mathrm{X}$, where $\varphi_{0}=\theta-\frac{\pi}{n}$, and $\mathrm{D}=r\left(\cos \left(\theta-\frac{\pi}{n}\right)-\cos \varphi\right)$. 


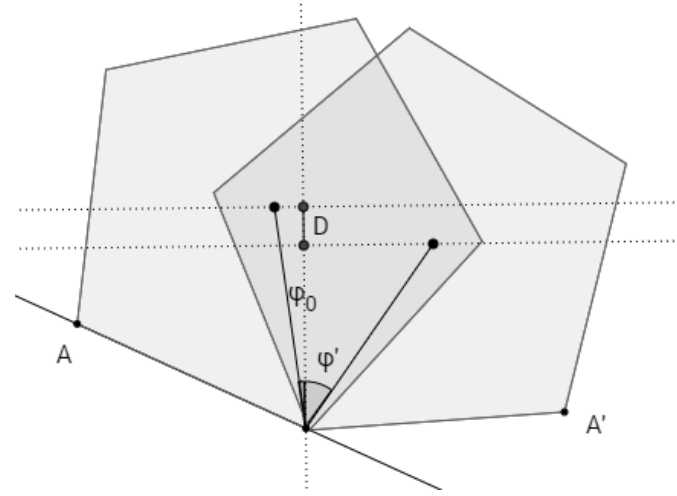

Fig X

\section{Derivation of Friction Coefficient}

The friction coefficient is determined by slowly raising the inclined plane until the prism that is initially at rest starts to slide; the prism is placed with its longer edge parallel to the ramp as shown in Fig XI, and the angle $\theta$ at which the prism slides is recorded.

Let the friction coefficient be $\mu_{f}$,

$$
\begin{gathered}
F_{f}=\mu_{f} F_{N}, \\
F_{N}=g_{y}=F_{g} \cos \theta=m g \cos \theta .
\end{gathered}
$$

At the instant before the prism slides, $\mathrm{F}_{f}=g_{x}=m g \sin \theta$; therefore,

$$
\begin{gathered}
\mu_{f} m g \cos \theta=m g \sin \theta \\
\mu_{f}=\frac{\sin \theta}{\cos \theta}
\end{gathered}
$$

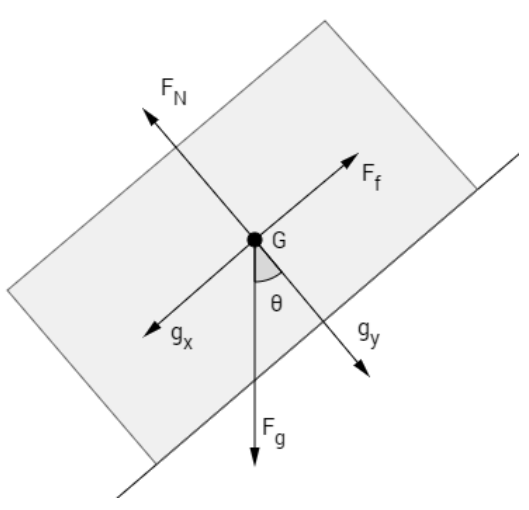

Fig XI 